\title{
A Case of Intractable Hiccups Successfully Treated with Pregabalin
}

\section{TO THE EDITOR}

Hiccups are involuntary, repetitive, and rhythmic, spasmodic contractions of the diaphragm. Generally, hiccups continuing for less than one day are considered as acute, with continuation more than a month defined as intractable (1). Pregabalin is a structural analog of gamma-aminobutyric acid (GABA), which binds to the alpha-2-delta subunit of $\mathrm{N}$-type calcium channels, resulting in decreased release of several neurotransmitters, including glutamate, noradrenalin, serotonin, dopamine, and substance $P(2-3)$. Pregabalin is one of the newer drugs approved as an anticonvulsant and for the treatment of neuropathic pain (4), and is classified as an adjuvant drug according to the World Health Organization analgesic ladder.

We present a case of idiopathic intractable hiccups managed using pregabalin.

A 65-year-old man initially visited a general practice clinic with hiccups. These hiccups continued for 3 years, with increasing frequency and intensity. The cause of the hiccups was examined. Magnetic resonance imaging (MRI) of the brain and computed tomography (CT) of the chest and abdomen did not provide any clinically useful information regarding the cause of the hiccups, and idiopathic hiccup was diagnosed. Several medications, used as monotherapy for his hiccups, were comprised of clonazepam, amitriptyline, haloperidol, chlorpromazine, metoclopramide, and Shakuyakukanzoto. In addition, the patient tried to breathe into a paper bag. However, the frequency and intensity of the hiccups did not decrease, preventing continuous sleep. The patient was therefore introduced to ourp clinic with intractable hiccups, 3 years after the first medical examination. When he visited us, he kept hiccupping.

We tried a cervical epidural block at C7-Th1 using the median approach and injected $5 \mathrm{~mL}$ of $0.5 \%$ lidocaine, which caused cessation of the hiccups after several minutes. However, the hiccups recurred after 8 - 9 hours. We then tried right and left phrenic nerve blocks, but the effects were again temporary. Oral baclofen was prescribed at $20 \mathrm{mg} /$ day, but the frequency of the hiccups did not decrease. With the aim of achieving a permanent cure for the hiccups, we decided to again change the pharmacotherapy. Treatment with baclofen was therefore changed to oral pregabalin at $150 \mathrm{mg} /$ day. Frequency and intensity of the hiccups decreased. When the dose of pregabalin was increased to $375 \mathrm{mg} /$ day, the hiccups stopped.

The hiccup is an involuntary medullary reflex of the chest wall. The reflex mechanism is based on the afferent pathway (the phrenic nerve, vagus nerve, and sympathetic chain arising from T6 to T12), the efferent pathways (phrenic nerves to the diaphragm, the intercostal nerves to the intercostal muscles, the scalenus anticus nerve to the scalene muscles, and the recurrent laryngeal nerve to the glottis), and the central component (brainstem connections between $\mathrm{C} 3$ and $\mathrm{C} 5$ linking the respiratory center, phrenic nerve nuclei, medullary reticular formation, and hypothalamus) (5). Disturbance of any one of these reflex components may result in intractable hiccups. A few reports in the literature have described persistent intractable hiccups due to lesions located in the medulla oblongata resulting from causes such as stroke, multiple sclerosis, and brain tumor (6-9). The patient we encountered was examined using MRI of the brain and whole-body CT, but the cause of the hiccups was not found, and idiopathic intractable hiccups were therefore diagnosed. Several nerve blocks, such as cervical epidural block, phrenic nerve block and stellate ganglion block, and pharmacotherapies such as clonazepam, tricyclic antidepressant, haloperidol, chlorpromazine, metoclopramide, and baclofen, are considered effective for hiccups $(10,11)$. In addition, gabapentin also reportedly has beneficial effects against intractable hiccups (12). Various mechanisms were proposed for the effects of gabapentin for the hiccup. They include the increase of endogenous GABA-mediated inhibition of inspiratory muscles, the decrease of calcium influx via inhibition of voltage-operated calcium channels in the presynaptic terminals of respiratory muscles, and the increase of levels of serotonin (12). Moreover, a case has been reported in which hiccups attributed to stroke were effectively treated using pregabalin (13). Pregabalin may be effective against intractable hiccups for the same reasons as for gabapentin. Dizziness, somnolence, and peripheral edema are the most frequent side effects of pregabalin (14), but no side effects were 
seen in this case.

In summary, idiopathic intractable hiccups were successfully treated in a patient we encountered using pregabalin.

Yuka Matsuki, MD

Department of Anesthesiology \& Reanimatology

University of Fukui

Faculty of Medicine Sciences

23-3 Matsuokashimoaizuki

Eiheijicho, Yoshidagun

Fukui 910-1193, Japan

E-mail: ymatsuki@u-fukui.ac.jp
Maki Mizogami, MD

University of Fukui

Faculty of Medicine Sciences

23-3 Matsuokashimoaizuki

Eiheijicho, Yoshidagun

Fukui 910-1193, Japan

E-mail:makikai@u-fukui.ac.jp

Kenji Shigemi, MD

University of Fukui

Faculty of Medicine Sciences

23-3 Matsuokashimoaizuki

Eiheijicho, Yoshidagun

Fukui 910-1193, Japan

E-mail: kshigemi@u-fukui.ac.jp

\section{References}

1. Alonso-Navarro H, Rubio L, Jiménez-Jiménez FJ. Refractory hiccup: Successful treatment with gabapentin. Clin Neuropharmacol 2007; 30:186-187.

2. Rosenstock J, Tuchman M, LaMoreaux L, Sharma U. Pregabalin for the treatment of painful diabetic peripheral neuropathy: A double-blind, placebo-controlled trial. Pain 2004; 110:628-638.

3. Smith HS, Harris R, Clauw D. Fibromyalgia: An afferent processing disorder leading to a complex pain generalized syndrome. Pain Physician 2011; 14:E217-E245.

4. Gilron I. Gabapentin and pregabalin for chronic neuropathic and early postsurgical pain: Current evidence and future directions. Curr Opin Anaesthesiol 2007; 20:456-472.

5. Hansen BJ, Rosenberg J. Persistent postoperative hiccups: A review. Acta Anaes- thesiol Scand 1993; 37:643-646.

6. Amirjamshidi A, Abbassioun K, Parsa K. Hiccup and neurosurgeons: $A$ report of 4 rare dorsal medullary compressive pathologies and review of the literature. Surg Neurol 2007; 67:395-402.

7. Funakawa I, Hara K, Yasuda T, Terao A. Intractable hiccups and sleep apnea syndrome in multiple sclerosis: Report of two cases. Acta Neurol Scand 1993; 88:401-405.

8. Musumeci A, Cristofori L, Bricolo A. Persistent hiccup as presenting symptom in medulla oblongata cavernoma: A case report and review of the literature. Clin Neurol Neurosurg 2000; 102:13-17.

9. Nagayama T, Kaji M, Hirano H, Niiro M, Kuratsu J. Intractable hiccups as a presenting symptom of cerebellar hemangioblastoma. Case report. J Neurosurg 2004; 100:1107-1110.
10. Sato S, Asakura N, Endo T, Naito H. Cervical epidural block can relieve postoperative intractable hiccups. Anesthesiology 1993; 78:1184-1186.

11. Choi TY, Lee MS, Ernst E. Acupuncture for cancer patients suffering from hiccups: A systematic review and meta-analysis. Complement Ther Med 2012; 20:447-455.

12. Ong AM, Tan CS, Foo MW, Kee TY. Gabapentin for intractable hiccups in a patient undergoing peritoneal dialysis. Perit Dial Int 2008; 28:667-668.

13. Vandemergel $X$, Mbeufet $M$, Renneboog B. Intractable hiccups successfully treated with pregabalin. Eur J Intern Med 2006; 17:522.

14. Matsuki $Y$, Morikawa $M$, Nishimoto $T$, Yasuda Y, Shigemi K. Heart failure associated with pregabalin. Pain Physician 2012; 15: E536-E537. 